\title{
Risk Analysis and Route Optimization of Dangerous Goods Transportation Based on the Empirical Path Set
}

\author{
Haixing Wang $\mathbb{D}$ and Qianqian Liang $(\mathbb{D}$ \\ School of Traffic \& Transportation, Beijing Jiaotong University, Beijing 100044, China \\ Correspondence should be addressed to Haixing Wang; hxwang@bjtu.edu.cn
}

Received 29 April 2020; Revised 3 June 2020; Accepted 15 June 2020; Published 9 July 2020

Academic Editor: Zhang Ronghui

Copyright (C 2020 Haixing Wang and Qianqian Liang. This is an open access article distributed under the Creative Commons Attribution License, which permits unrestricted use, distribution, and reproduction in any medium, provided the original work is properly cited.

\begin{abstract}
Based on the empirical path set generated from the track data of dangerous goods vehicles, we present a new method for the risk analysis and path optimization of dangerous goods transportation. First of all, by exploring the travel rules of dangerous goods transport vehicles hidden in the track data, combined with the path set generation algorithm, the method of determining the empirical path set of dangerous goods transport is studied. Secondly, based on the empirical path set, mainly considering the travel rules of vehicles and people on the road, as well as the distribution of population and environment-sensitive areas along the road, a dual objective path selection model is proposed to comprehensively measure the risk and cost of road transportation under timevarying conditions. On this basis, given the principle of avoiding high-risk transportation paths, a comprehensive method of integrating multiple algorithms is proposed to solve the model. Finally, taking a road network as an example, the practicability and effectiveness of the proposed method are verified. The method proposed takes both practicability and safety into account. Based on the experience path set, considering the time-varying characteristics, the decision-maker could choose the appropriate transportation path of dangerous goods according to different preferences, so as to better solve the problem of path selection for dangerous goods transportation.
\end{abstract}

\section{Introduction}

Dangerous goods transportation is featured in the location moving, complicated and changing natural environment, and close correlation with the social life of the human. Any accident in transportation, which is more random than those occurring in the production, may give rise to more serious consequences.

Existing researches on dangerous goods transportation mainly concern the analysis of transportation risk or the selection of transportation path. Many scholars combine these two aspects to optimize the transportation path to lower risks in the transportation path. Inanloo and Tansel combine Gaussian Model and ArcGIS to calculate the risk of the exposed population, risk of the environment-sensitive area, time delay cost, and link transportation cost along the transportation path with consideration to the atmospheric stability [1]. Dadkar et al. solve the path selection problem taking into account the stochastic changes of each highway facility performance as time goes by based on the $k$-shortest path algorithm [2]. Dolphin et al. describe a software suite designed to perform sensitivity and cost/benefit analyses on selected transportation paths and vehicles to minimize the risk associated with the shipment of dangerous goods [3]. $\mathrm{Ma}$ et al. utilize the risk assessment matrix to assess the integrated risk of the link from perspectives of the probability consequence level and the consequent risk level and then acquire the risk in the transportation path by the weighted distance method [4].

Some scholars optimize the transportation path of dangerous goods by multiobjective decisions to meet the demand for safety and economic efficiency. Junior and Márcio De Almeid present an application of the modal choice method (MCM) to establish an order of priority among the alternatives considered to carry a determined hazardous product [5]. Samanlioglu developed a new 
multiobjective location-routing model in which three criteria were considered: minimizing the total transportation cost, minimizing the cost of dangerous goods transportation, and minimizing total transportation risk associated with the population exposure along transportation paths and around treatment [6]. In the multiobjective selection of hazmat transportation path in an urban road network, Pamučar et al. proposed a new cost and risk evaluation method by using an adaptive neural fuzzy inference system (ANFIS) [7]. Mahmoudabadi and Zhang defined the transportation risk as a chaotic variable, used two methods to establish a chaotic model, and analyzed the results of different sizes of risks and costs combined with cases. The results show that the path of chaos model makes the frequency of path selection change with different cost and risk priorities converging to the logical mapping equation [8]. Considering that the potential high risk in dangerous goods transportation does not lead to high return, Kwon introduced CVAR (conditional value at risk) model into the optimization model of the transportation path of dangerous goods [9]. Jassbi and Makvandi established a multiobjective optimization model with the objectives of the transportation distance of dangerous goods transport vehicles, the population coverage rate along the transportation path, and the risk of the road section. They solved it through a genetic algorithm [10].

Accident rate and severity analysis is also an important aspect of this study. Haghighi et al. examined the impact of a wide range of roadway geometric features on the severity outcomes of crashes that occurred on rural two-lane highways [11]. Zeng et al. investigated the inclusion of spatiotemporal correlation and interaction in a multivariate random-parameters Tobit model and their influence on fitting areal crash rates with different severity outcomes [12]. Zeng et al. presented an empirical investigation of the impacts of real-time weather conditions on the freeway crash severity [13]. Zeng et al. presented a joint analysis of daytime and nighttime crash frequencies at the zone level with consideration of spatial correlations [14]. Imprialou and Quddus reviewed current literature on the state of crash data quality for each of the questions separately [15].

Along with the development of ITS (Intelligent Transport System), the traveler will select and adjust the path appropriately, given the traffic information. As a result, the path selection model under time-varying conditions may be more practical. Pradhananga et al. proposed a transportation path selection model considering the time window after analyzing the waiting time in the dangerous goods transportation and solved it by genetic algorithm [16].

However, most of the above research mainly focuses on the optimization of path selection based on different models and algorithms, less on the empirical path used in the actual dangerous goods transportation. Nowadays, in China and many countries around the world, more and more dangerous goods vehicles are monitored, and a large number of actual data on dangerous goods transportation are accumulated. For example, by using the track data of dangerous goods vehicles, it is more convenient to generate the path of dangerous goods vehicles, and it can also be beneficial for analyzing the travel time according to the time interval recorded by the data.

These data, which reflect the operating experience of the drivers of dangerous goods vehicles, have important reference value for the paths' selection for dangerous goods transportation and could be used to conduct a more reasonable path set. Although errors are easily produced for path selection analysis based on independent individuals, with the increase of samples, the commonness for groups becomes apparent. To complete the task faster and better, the drivers of dangerous goods vehicles will choose the reasonable path according to the experience as much as possible, which reflected in the data the fact that most drivers will choose a similar path set, that is, the empirical path set. We could get the empirical path set by analyzing the rich experience of drivers for dangerous goods transportation. It has a great advantage to use the path set based on the driver's experience, and also it makes the work more reasonable and practical usability.

Therefore, to improve the feasibility and applicability of dangerous goods operation path, this paper proposes an empirical path set generation method based on the track data of dangerous goods vehicles. We could get the empirical path set by careful study of the track distribution map and the heat map. By making a thorough inquiry for the rule hidden in the track data, combined with the path set generation algorithm, the empirical path set for dangerous goods transportation could be formed. At the same time, in dealing with the problem of less consideration of safety for the empirical path set, the model and algorithm considering the influence factors for safety during the process of dangerous goods transportation are designed. Based on the comprehensive analysis of the traffic flow on the road and the distribution of population and environment-sensitive areas along the road, the time-varying risk of the road section is evaluated, and the double objective optimization model combined with the transportation cost and risk under the time-varying conditions is established, so as to optimize the path selection for dangerous goods transportation. The method proposed in this paper takes both practicability and safety into account, and it is beneficial for better solving the problem of path selection for dangerous goods transportation. The flow chart based on the proposed research work is shown in Figure 1.

After the generating strategy for the empirical path set is given in Section 1, Section 2 conducts the process of quantitative risk analysis. Then, a description of the path selection optimization process using an appropriate riskcost function under time-varying conditions is presented in Section 3, followed by a solution method that combined improved DFS algorithm with fast nondominated sorting, which is described in Section 4. Finally, in Section 5, the proposed model and algorithm are verified through a design case.

\section{Strategy for Empirical Path Set Generating}

2.1. Network Joint Control System for Dangerous Goods Transportation. To strengthen the management and 


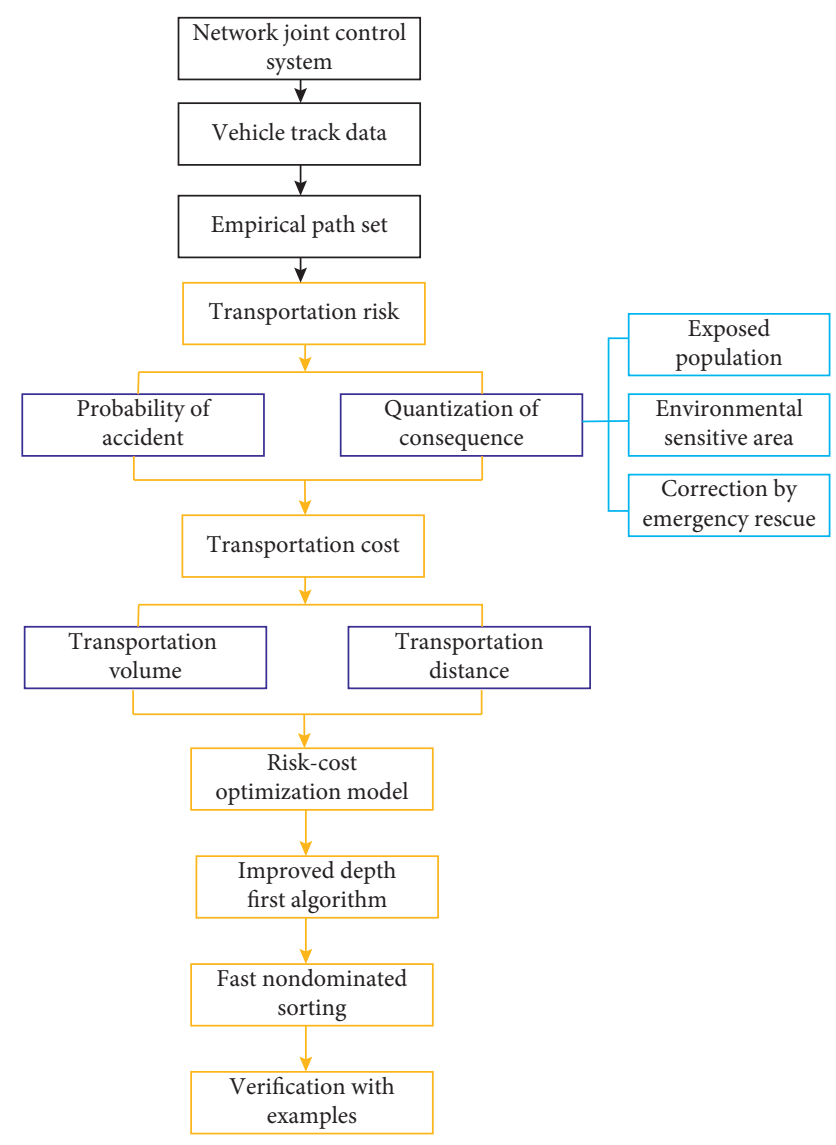

Figure 1: The flow chart $t$ based on the proposed research work.

improve the safety level of dangerous goods transportation, China has established a joint control system for dangerous goods transportation based on satellite positioning technology. By the beginning of 2020, the ratio of vehicles joining the network has exceeded $99.7 \%$. The organizational structure of the network joint control system is shown in Figure 2 [17].

\subsection{Handling of Abnormal Track Data of Dangerous Goods} Vehicles. When using the network joint control system to collect traffic information, the on-board equipment continuously queries and obtains the real-time data of vehicles and then sends it to the computing center, so as to get the real-time operation status of dangerous goods vehicles in each road section. In the actual operation process, due to the influence of equipment, communication mode, and random error, some abnormal data may be produced. To get accurate information, it is necessary to identify and selectively repair the anomalous data. At the same time, it is also required to filter the data to eliminate the invalid data or reduce noise interference, so as to improve the quality of the data.

\subsubsection{Data Rationality and Integrity Verification.} Because the on-board equipment needs to collect data at timed intervals, and it is easy to be interfered with by strong signals from the outside, it may produce data loss and error.
Therefore, it is necessary to check the rationality and integrity of the track data. As to the occurrence of continuous wrong data or data loss (such as constant data loss caused by communication failure of vehicle terminal), to ensure the accuracy of collected data, some of the fault data could be removed. When there are only some wrong data, the historical trend method and time series method could be used to repair or supplement the fault data selectively.

2.2.2. Data Sifting and Filtering. A large number of data could be obtained from the vehicle monitoring system. But too much data could make the system overload and result in the unnecessary increase in equipment cost. Therefore, reasonable data sifting and elimination of some invalid data will not only affect the results but also improve the efficiency.

In the operation process, the data may be affected by road conditions, vehicle performance and drivers' habits, and other factors, and a large amount of random data could be generated. For the random components contained could create a wrong impression of the data application, it is better to filter the data. The principle of the filtering method is not to change the rule of the original data and remove the random components. The standard filtering methods include median filtering, moving average filtering, exponential filtering, and Kalman filtering.

\subsection{Method of the Empirical Path Set Generating}

2.3.1. Identification of Starting and Ending Points. In the network joint control system, the original information of the track data for dangerous goods vehicles mainly includes latitude, longitude, time, and speed. Through the analysis of the data, the system will continuously collect the vehicle operation data. Still, the vehicle is not always running, and there may be dwell points. It is necessary to judge these stops further and then identify whether these stops are shortstops caused by traffic jams, waiting at intersections, refueling, or midway rest during the driving process or stops before and after the trip. Through the analysis of the stop point, the starting and ending points and the running track could be determined.

2.3.2. Map Matching. The function of map matching is to connect the vehicle location tracks with the road in the digital map and then determine the position of the vehicle on the map. The basic idea is to select a certain number of points or curves on the map and get a pair of samples to match them. Finally, the vehicle in motion is located, and the positioning error is reduced by map matching. Therefore, map matching could not only reduce the error of location data and improve the accuracy of location data but also achieve a more intuitive and visual effect.

2.3.3. Empirical Path Set Based on Track Distribution Diagram and Heat Diagram. Firstly, the paths of the dangerous goods vehicles in the study area are marked out, and the track distribution diagram of the vehicles could also be 


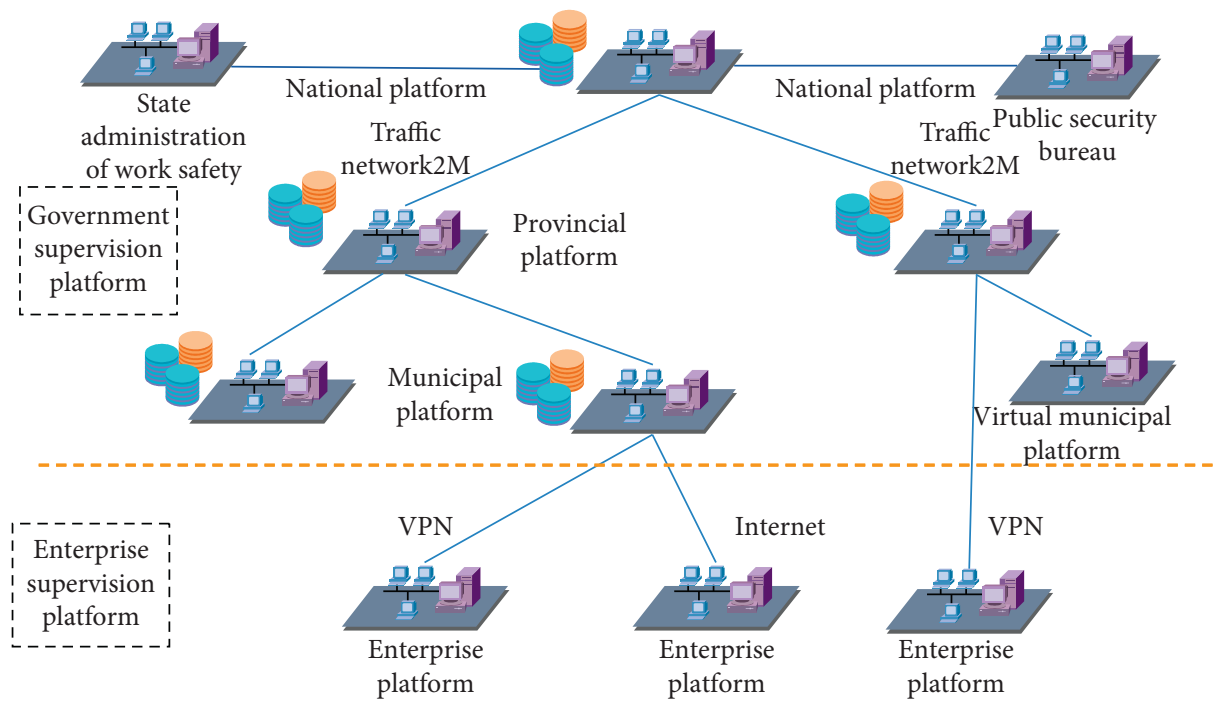

FIgURE 2: The organizational structure of the network joint control system.

generated, as shown in Figure 3. The gradual change from blue to red indicates the difference in the density of the vehicles from low to high. The dangerous goods vehicle track map could be gridded, and the grid size is determined according to the density of the road and the view scale. Because the density of the vehicles on each road is different, the grid may have different densities. The grid with more significant density will be used for the areas with deep color, and the grid with smaller density will be used for the fields with a lighter color (Figure 4), so as to minimize the error.

Kernel density estimation may be used in density analysis, which could make full use of the original information of data and avoid the introduction of prior knowledge. Through density analysis, the points or lines could be transformed into continuous surfaces. Then, the data aggregation status of the whole area could be calculated by searching the areas where the points or lines are concentrated. At the same time, the density analysis will be in progress by selecting an appropriate radiation radius, and the heat diagram could be generated. The classification of the heat for dangerous goods vehicles is based on the color depth. The empirical path set of the vehicle could be obtained mainly based on the track distribution diagram and heat diagram.

\section{Quantitative Risk Analysis}

The risk may be defined as the loss or lost value of expectation. In the comprehensive risk assessment method, the risk is regarded as a kind of mathematic value of expectation lost by humans within a particular area. Therefore, we may define the leakage risk of dangerous goods as the product of the leakage probability and leakage consequence.

3.1. Determination of Probability of Leakage Accident. We may not acquire the probability of accident through the statistical data since statistics of transportation accidents of

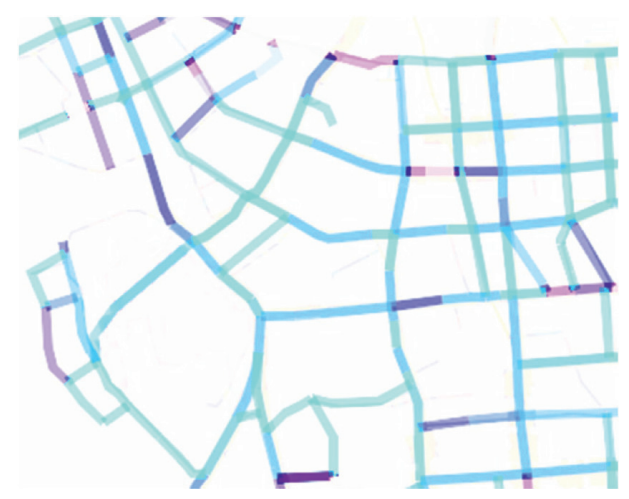

Figure 3: Vehicle track distribution diagram.

dangerous goods are absent in China. The quantitative probability of transportation accidents of dangerous goods declared by DuPont is $10^{-8} \sim 10^{-6}$. And the traffic accident rate comes from the traffic accident data of three major states of the USA and the traffic accident rate calculated based on Gross Domestic Product (GDP) and transportation situation [18], as demonstrated in Table 1. It is also indicated by the statistical analysis of 562 transportation accidents of tank trucks of dangerous goods that the conditional probability of leakage by tank trucks of dangerous goods due to traffic accidents is 0.86 [19].

At the same time, we also consider the impact of weather factors on the accident. Based on fine days, we multiply different weather conditions such as rainy days, snowy days, and foggy days by different coefficients to reflect the impact of weather on the accident. The weather coefficient is different in different areas. Take the region involved in the following example as a subject. The average number of different types of weather days, the average number of accidents under various types of weather, and the number of daily average accidents (certain types of weather) of the region within 5 years are shown in Table 2 and Figure 5. 

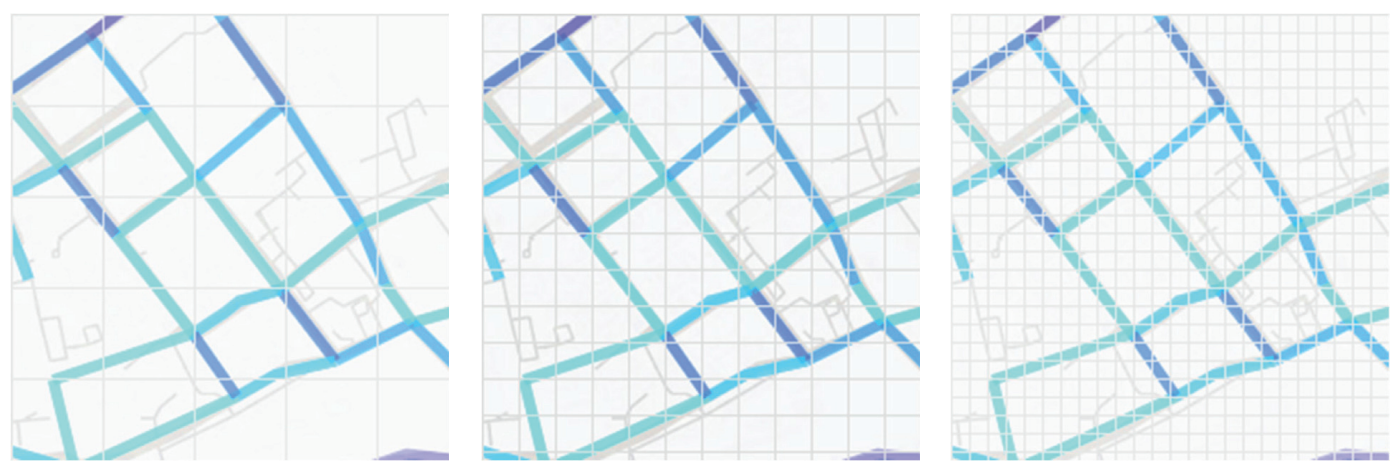

FIgURE 4: Gridding with different densities.

TABLE 1: Basic traffic accident rate of dangerous goods transportation.

\begin{tabular}{lccc}
\hline Area & Road class & Average truck traffic accident rate $\left(\right.$ once $/ 10^{8}$ veh.km) \\
\hline & Expressway, class I highway & & 4.485 \\
& Class II highway & & 6.512 \\
& Class III and IV highway & & 3.485 \\
Urban & Substandard highway & Double lane & 2.458 \\
& & Undivided lane & 3.925 \\
& Urban road & Double lane & 3.511 \\
& & Multilane & 5.058 \\
\hline & & One-way street & 3.935 \\
Rural & Expressway, class I highway & Undivided lane & 5.645 \\
& Class II highway & & 1.330 \\
& Class III and IV highway & & 1.646 \\
& Substandard highway & Double lane & 0.617 \\
\end{tabular}

TABLE 2: Coefficient between weather and accident.

\begin{tabular}{|c|c|c|c|c|}
\hline Item & Fine & Rain & Snow & Fog \\
\hline Number of accidents & 245 & 52 & 17 & 10 \\
\hline Weather days & 292 & 55 & 6 & 8 \\
\hline Number of accidents per day (certain type of weather) & 0.84 & 0.95 & 2.83 & 1.25 \\
\hline Weather coefficient & 1 & 1.13 & 3.37 & 1.49 \\
\hline
\end{tabular}

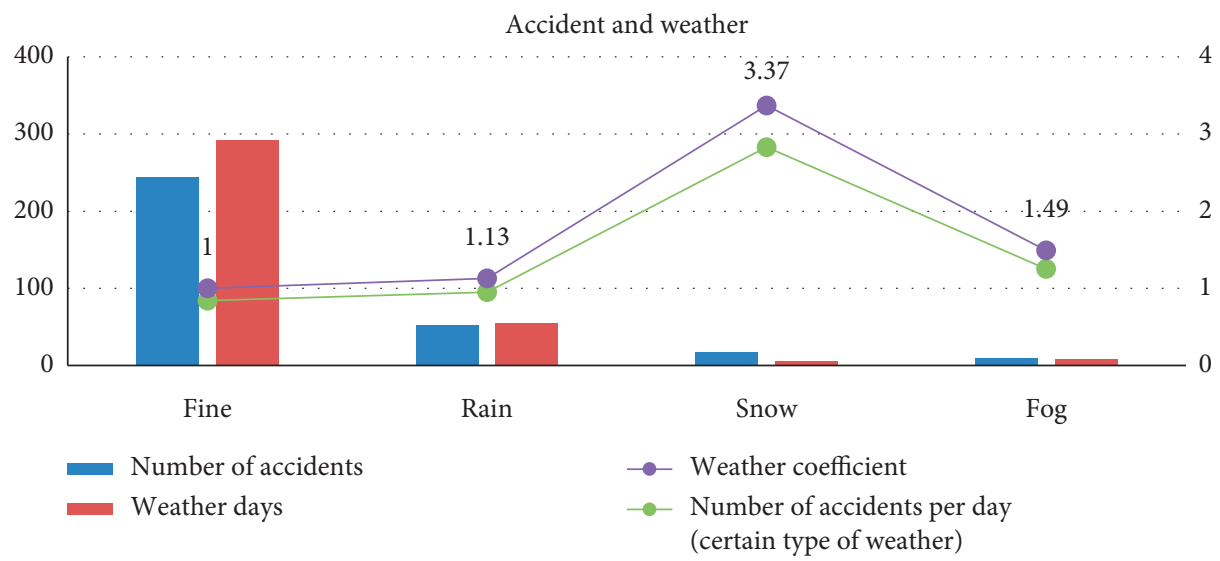

FIgURE 5: Coefficient between weather and accident. 
3.2. Quantization of Leakage Accident Consequence. The leakage of dangerous goods may pose a severe impact on the surrounding environment and result in various kinds of severe consequences. And the most common consequences are injury and death of exposed population and environmental disruption $[20,21]$. Hence, we calculate the value loss of leakage accidents under mixed traffic conditions from perspectives of exposed population and environmentally sensitive area.

3.2.1. Quantization of Loss of Exposed Population. We calculate the concentration of hazardous gas at different positions through the simulated calculation initially and then convert the probability to the corresponding death rate by the probability function method. The relation between probability $Y$ and the concentration of hazardous gas and the duration of the contact is demonstrated in

$$
Y=A+B \ln \left(C^{n} t\right)
$$

where $C$ is the concentration of hazardous gas in contact, $\times 10^{-6}$; $t$ is the duration of contact with hazardous gas, min; and $A, B$, and $n$ are constant parameters depending on the nature of hazardous gas.

Generally, the duration of contact with hazardous gas will be no longer than $30 \mathrm{~min}$, during which the people may escape from the site or take protective measures [22, 23].

The conversion between probability and death rate is demonstrated in Table 3, and the probability value is $0 \sim 9 \%$ $[24,25]$.

As different concentrations and durations of contact lead to different healthy influences and medical treatment expenses, determination of the risk of exposed population shall consider those suffering from different extents of injuries by contacting the toxic gas while considering those becoming dead by contacting the toxic gas. The economic loss of the population with different extents of injuries is demonstrated in Table 4.

We may quantify the risk of exposed population caused by one leakage accident by the death rate of different threshold areas derived from the above study.

3.2.2. Loss Calculation of Environmentally Sensitive Areas. Damage to the environmentally sensitive area brought by the leakage of dangerous goods mainly includes pollution of soil and water, destruction of animals and plants, and corrosion of cultural relics. We will assess the loss of environmentally sensitive areas caused by the leakage from the perspective of value loss.

We divide environmentally sensitive areas into four types, which are water area, forest land, agricultural planting area, and public green space. We determine the value of the environment-sensitive area; different assessment methods of value loss for different environmental sensitive areas are listed in Table 5. And then, we determine the loss brought to the sensitive area by different concentrations of leakage to recognize the converted area loss of each sensitive
TABle 3: Conversion between probability and death rate.

\begin{tabular}{|c|c|c|c|c|c|c|c|c|c|c|}
\hline$P(\%)$ & 0 & 1 & 2 & 3 & 4 & 5 & 6 & 7 & 8 & 9 \\
\hline 0 & & & 5 & 2 & 5 & 36 & 3.4 & 52 & 59 & 3.66 \\
\hline 10 & .72 & & 2 & & & & & & & 4.12 \\
\hline 20 & 6 & 4.19 & 3 & 26 & 4.29 & 33 & 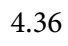 & 9 & 2 & 4.45 \\
\hline 30 & & & & & & 51 & & 67 & & 4.72 \\
\hline 40 & 75 & 4.77 & 4.80 & 4.82 & 4.85 & 4.87 & 4.90 & 4.92 & 4.95 & 4.97 \\
\hline 50 & 00 & 5.03 & 5.05 & 5.08 & 5.10 & 5.13 & 5.15 & 5.18 & 5.20 & 5.23 \\
\hline 60 & 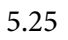 & 5.2 & 5.31 & 53 & 5.36 & 5.39 & 5. & 5.44 & 5.47 & 5.50 \\
\hline 70 & 2 & 5.55 & 5 & 5. & 5.64 & 5.67 & 71 & 74 & 5.77 & 5.81 \\
\hline 80 & 5.84 & 5.88 & 5.92 & 5.95 & 5.99 & 6.04 & 6.08 & 6.13 & 6.18 & 6.23 \\
\hline 90 & 6.28 & 6.34 & 6.41 & 6.48 & 6.55 & 6.64 & 6.75 & 6.88 & 7.05 & 7.33 \\
\hline \multirow[t]{2}{*}{99} & 0 & 0.1 & 0.2 & 0.3 & 0.4 & 0.5 & 0.6 & 0.7 & 0.8 & 0.9 \\
\hline & 33 & 37 & .41 & .46 & 7.51 & 7.58 & 7.58 & 7.65 & 7.88 & 8.09 \\
\hline
\end{tabular}

environmental area and finally transform the area loss to the value loss.

Value loss of the environmentally sensitive area may be expressed as

$$
W=\sum_{i=1}^{3} \delta_{i}\left(\sum_{j=1}^{5} V_{j} S_{i j}\right) .
$$

In the formula, $W$ is the total value loss of environmentally sensitive area within the area impacted by the leakage, $\delta_{i}$ is the risk impact weight of area $i, V_{j}$ is the cost per unit of the $j^{\text {th }}$ kind of environmentally sensitive area, and $S_{i j}$ is the area of the $j^{\text {th }}$ kind of environmentally sensitive area within area $i, \mathrm{~m}^{2}$.

\subsubsection{Correction of Accident Consequence by Emergency} Rescue. Emergency rescue is quite essential for lowering the accident consequence risk that shall be sufficiently concerned in the risk assessment [26]. In the evaluation of the leakage accident risk, the capability of emergency rescue is included in the risk assessment model as a kind of "offset."

ICI (Imperial Chemical Industries) Mond method notes the compensation coefficient towards the fire rescue in the evaluation of compensation to the unit danger. In this paper, the value-taking standard of the compensation coefficient is established in combination with the time for the Chinese fire rescue team to arrive at the site, as demonstrated in Table 6.

3.2.4. Risk Assessment Model. Transportation risk $R$ of the unit link $l$ at a particular moment may be expressed by

$$
\begin{aligned}
R(l)= & P_{\text {rel }}(l) \cdot\left(B^{\text {in }}\left(t_{x}\right)+B^{\text {our }}\left(t_{x}\right)+W\right) \cdot \tau \\
= & P_{t}(l) \cdot P_{t \longrightarrow \text { rel }}(l) \cdot\left[\sum_{i=1}^{3} \sum_{k=1}^{3} S_{i}^{\text {in }} d_{i}^{\text {in }}\left(t_{x}\right) P_{i k} N_{k}\right. \\
& \left.+\sum_{i=1}^{3} \sum_{k=1}^{3} S_{i}^{\text {out }} d_{i}^{\text {out }}\left(t_{x}\right) P_{i k} N_{k}+\sum_{i=1}^{3} \delta_{i}\left(\sum_{j=1}^{4} V_{j} S_{i j}\right)\right] \cdot \tau .
\end{aligned}
$$


TABLE 4: Statistical analysis of mean economic loss of one person/time in an accident.

\begin{tabular}{|c|c|c|c|c|c|}
\hline Injury type & Slight injury & Partial disability & Serious disability & Total disability & Death \\
\hline Economic loss (RMB10,000) & 0.05 & 0.3 & 2.0 & 8.0 & 20.0 \\
\hline
\end{tabular}

TABle 5: Assessment methods of value loss for different environmental sensitive areas.

\begin{tabular}{lccc}
\hline Sensitive area & Assessment method & Calculation formula & Parameter \\
\hline Water area & Replacement cost & $V=\sum_{n} P_{n}$ & $P_{n}:$ restoration cost of the water body \\
Forest land & Shadow project & $V=P_{p} \cdot S$ & $P_{P}:$ construction cost per unit area of forest land \\
Agricultural planting area & Market value & $V=\sum \Delta m_{i} \cdot P_{i}$ & $\begin{array}{c}\Delta m_{i}: \text { cut-down yield of crops } \\
\text { Public green space }\end{array}$ \\
\hline
\end{tabular}

TABLE 6: Value-taking standard of emergency rescue team compensation coefficient $\tau$.

\begin{tabular}{lcc}
\hline Equipment and level of fire department & Response time & Compensation coefficient $\tau$ \\
\hline 1 fire engine & $\leq 10 \mathrm{~min}$ & 0.90 \\
2 fire engines & $\leq 10 \mathrm{~min}$ & 0.85 \\
3 fire engines & $\leq 10 \mathrm{~min}$ & 0.80 \\
4 fire engines & $\leq 10 \mathrm{~min}$ & 0.75 \\
5 or more fire engines & $\leq 10 \mathrm{~min}$ & 0.7 \\
\hline
\end{tabular}

\section{Path Selection under Time- Varying Conditions}

4.1. Analysis of Influencing Factors of Path Selection. Dangerous goods constitute a considerable threat to the production and living of human beings, as well as the natural environment due to its features of toxicity, inflammability, and explosion hazard, so the selection of transportation paths must concern the safety while considering the transportation cost.

Erkut points out that when the dangerous goods are transported in the road network, the risk and cost of transportation will never achieve the optimal status simultaneously [27, 28]. The transportation enterprise tends to pursue a lower transportation cost while the government requires the minimum transportation risk. Therefore, a path concerning both cost and risk of transportation must be selected for dangerous goods transportation.

\subsection{Calculation of Transportation Cost under Time-Varying} Conditions. Usually, the transportation cost is closely related to transportation mileage and load. The transportation cost mainly depends on the transportation volume $Q$ and transportation distance $L$. The author sets the road network as $G(N, A)$, in which $N$ represents the node. $A$ represents the set of edges, establishes the link as $(i, j)$, and sets the transportation cost of dangerous goods $k$ as $c^{k}$ (yuan $\left./(\mathrm{kg} \cdot \mathrm{km})\right)$, then $i, j \in N,(i, j) \in A$, and the total cost $C^{k}$ of a particular transportation path may be expressed as

$$
C^{k}=\sum_{(i, j) \in A} c_{i j}^{k}=\sum_{(i, j) \in A} c^{k} Q^{k} l_{i j}
$$

The speed of different vehicles in the mixed traffic differs from each other mainly in the actual transportation, giving rise to a relatively significant transverse interference, thus restraining the running speed of motor significantly and leading to the congestion of links indirectly. It has been indicated in relevant surveys that the increase in transportation cost is basically in a linear relationship with the link congestion.

The manifestation of link congestion in the transportation cost shall refer to the BPR function proposed by the Bureau of Public Roads of the USA:

$$
t_{i j}=t_{0}\left[1+\alpha\left(\frac{q_{i j}}{c_{i j}}\right)^{\beta}\right]
$$

In formula (5), $t_{i j}$ is the time impedance on the link $(i, j)$; $t_{0}$ is the zero-flow impedance; $q_{i j}$ is the traffic volume on the link $(i, j) ; c_{i j}$ is the actual traffic capacity of the link $(i, j) ; \alpha$ and $\beta$ are the retardation factors, whose values taken by the Bureau of Public Roads of the USA are $\alpha=0.15$ and $\beta=4$. Transportation costs concerning traffic congestion under time-varying conditions in the mixed traffic may be expressed by

$$
\begin{aligned}
C^{k}= & \sum_{(i, j) \in A} c_{i j}^{k}\left(t_{x}\right)=\sum_{(i, j) \in A} c^{k}\left(t_{x}\right) Q^{k} l_{i j}=\sum_{(i, j) \in A} c^{k} \\
& \cdot\left[1+\alpha\left(\frac{q_{i j}\left(t_{x}\right)}{c_{i j}}\right)^{\beta}\right] Q^{k} l_{i j}, \\
q_{i j}\left(t_{x}\right)= & \sum_{y=1}^{4} q_{i j}^{y}\left(t_{x}\right) \cdot \xi .
\end{aligned}
$$

In formula (6), $c_{i j}^{k}\left(t_{x}\right)$ is the transportation cost of dangerous goods $k$ on the link $(i, j)$ at the moment $t_{x} ; q_{i j}\left(t_{x}\right)$ is the standard equivalent traffic volume on the link $(i, j)$ at the moment $t_{x} ; y=1,2,3,4$, representing the nonmotor 
vehicle, sedan, midsize vehicle, and oversize vehicle, respectively; $\xi$ is the vehicle conversion coefficient where we take the sedan as the standard vehicle conversion coefficient in this paper.

4.3. Establishment of Transportation Risk-Cost Optimization Model under Time-Varying Conditions. Firstly, two groups of binary decision variables are defined:

$$
x_{i j}=0 \text { or } 1,
$$

$x_{i j}=1$ if there is any transportation task between node $i$ and the next node $j ; x_{i j}=0$, otherwise;

$$
y_{i j}=0 \text { or } 1,
$$

$y_{i j}=1$ if the vehicle meets requirements on weight limits between node $i$ and node $j ; y_{i j}=+\infty$, otherwise.

The establishment of the path selection model under timevarying conditions to pursue the minimum transportation risk and the lowest transportation cost is expressed by

$$
\begin{aligned}
& \min R^{k}=\sum_{i, j \in A} x_{i j} y_{i j} R_{i j}^{k}\left(i, j, t_{i}\right), \\
& \min C^{k}=\sum_{i, j \in A} x_{i j} y_{i j} c_{i j}^{k}\left(i, j, t_{i}\right),
\end{aligned}
$$

s.t.

$$
\sum_{j=1}^{n} x_{i j}-\sum_{j=1}^{n} x_{j i}=\left\{\begin{array}{l}
1, \\
-1, \\
0 .
\end{array}\right.
$$

It is equal to 1 if $i$ is the starting point, -1 if $i$ is the end point $\forall i \in N$, and 0 , else:

$$
\begin{gathered}
y_{i j}= \begin{cases}1, & q(i, j) \geq m(i, j), \\
+\infty, & q(i, j)<m(i, j),\end{cases} \\
\sum_{j} x_{i j} \leq 1, \quad \forall i, j \in N, \\
x_{i j} \in\{0,1\}, \quad \forall i, j \in N, \\
y_{i j} \in\{1,+\infty\}, \quad \forall i, j \in N .
\end{gathered}
$$

In formula (14), $R_{i j}^{k}\left(i, j, t_{i}\right)$ and $c_{i j}^{k}\left(i, j, t_{i}\right)$ represent the fact that, in the dangerous goods transportation $k$, the transportation risk and cost on the link $(i, j)$ correspond to the moment of entering the current node $i$. If the corresponding cost matrix and risk matrix vary along with the time change when passing link $(i, j)$, then the value corresponding to the moment of entering node $i$ shall prevail. $q(i, j)$ represents the weight limit of the link $(i, j)$ while $m(i, j)$ represents the sum of the self-weight and actual load of the tank truck.

Constraint condition (10) restricts the flow balance of the node, constraint condition (11) is the weight limit of the link, and constraint conditions (12)-(14) are binary decision variables.

\section{Solution Method}

There may be conflict or incomparability among several objectives in the problems of multiobjective planning. One solution may be the best for a particular objective. Still, it may be disadvantageous for another, and the attributes of each link within the road network may vary along with the time to complicate the solution procedure. Since intelligent algorithms like S.A. and C.A. may fail to acquire the globally optimal solution of the multiobjective function due to improper settings of parameters, the multiobjective function is solved by the Depth-First Search from the graph theory.

We search for feasible solutions by DFS algorithm through Python programming and then set constraint conditions and delete inferior solutions directly to acquire feasible solutions. Then, we conduct the fast nondominated sorting of feasible solutions to obtain the Pareto nondominated solution set for the decision-maker to select the transportation path by the practical demand.

5.1. Improved DFS Algorithm. Depth-First Search is a kind of graph-theoretical algorithm [29] with the following necessary steps:

Step 1: mark the initial node as the current point and then turn to Step 2

Step 2: search each neighboring node, extend and mark node if it has not been visited and take as the current point, and repeat Step 2; if all the adjacent nodes have been visited, then return to the parent node and make the parent node as the current node, and repeat Step 2

Step 3: finish when the current node is the initial node, and all the neighboring nodes have been visited.

Based on the DFS algorithm, we add constraint conditions in combination with the actual situation of the road network and our advanced understanding and judgment of the road network to prevent the algorithm from making unnecessarily repeated searches and entering the rotating ring, so as to shorten the calculation time.

5.2. Fast Nondominated Sorting. Pareto proposed the concept of the nondominated solution of multiobjective function in 1986, a set theory-based vector evaluation method of multiobjective function. There is no advantage or disadvantage of the nondominated solution as for all the objective functions, so the nondominated solution is also called Pareto optimal solution.

Pareto dominance relation: in the minimal multiobjective problem, the author assumes a vector $\bar{F}(\bar{x})=\left(F(\bar{x}), F_{2}(\bar{x})\right), \ldots$, comprising $n$ objective functions and $n$ objective components $F_{i}(i=1, \ldots, n)$, and randomly sets two decision variables $\bar{x}_{a}, \bar{x}_{b} \in U$, and their decisionmaking relationship is shown in Figure 6.

Dominated sorting method is widely applied to the genetic algorithm. The purpose of nondominated sorting is to acquire a nondominated solution. The collection of nondominated solutions is the so-called nondominated 


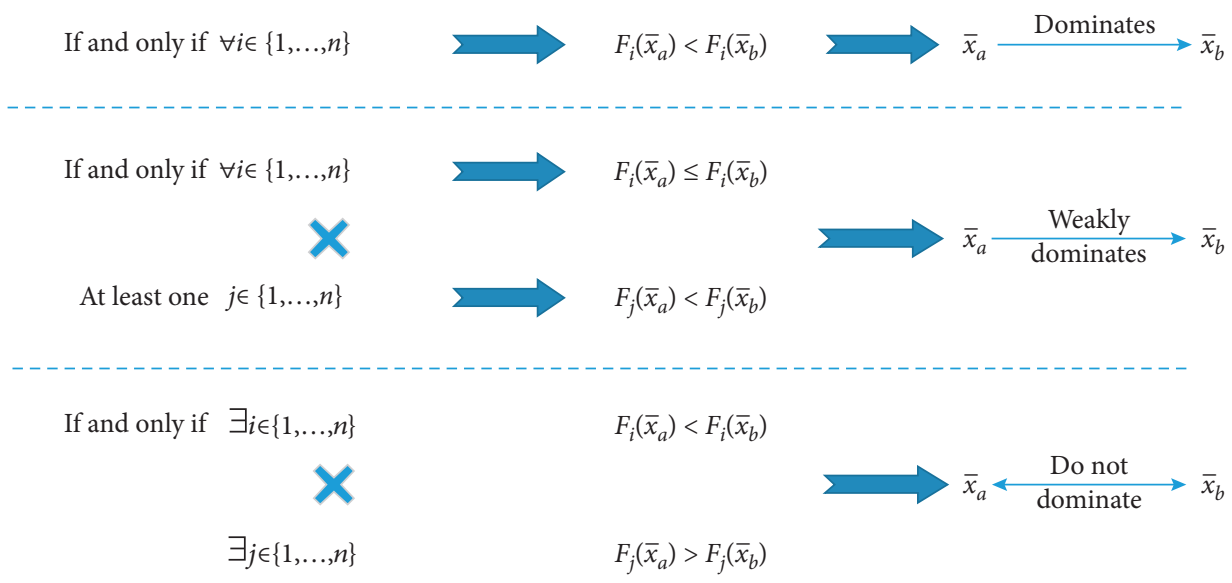

FIGURE 6: Decision-making relationship.

solution set in which the decision-maker may select the solution meeting the practical demand from such a nondominated solution set [30].

\section{Analysis of Example}

6.1. Road Network. The problem studied in this paper is of large scale, involving a large number of data, and it is difficult to obtain all the actual data, so we choose a relatively largescale calculation example with 36 nodes and 64 links for the feasibility and rationality of the algorithm. Road network of City A is taken as the object, which is simply demonstrated in Figure 7, and altogether 36 nodes and 64 links are selected for the analysis. The crossing point not marked as a node stands for the plane separation in the form of an overpass where there is no actual intersection.

6.2. Transportation Risk and Cost of the Link under TimeVarying Conditions. The continuous leakage by a tank truck loading 15 tons of liquid nitrogen is taken as an example to consider the risk calculation method in the mixed traffic flow under time-varying conditions introduced. In the beginning, we set the parameters of the accident scenario and combine the variety of mixed traffic flow of the link (flow of pedestrians and nonmotor vehicles) and the distribution of population density and various kinds of environment-sensitive areas along the path to calculate the risk of each elemental link in the road network to acquire the transportation risk of each link in different time frames [31].

Regarding the ton oil expense in the phased freight settlement mode introduced by Chen Gang, transportation cost of liquid nitrogen is Chinese Yuan (CNY) $0.765 /($ ton $\cdot \mathrm{km})$; the author calculates the transportation cost of the link in each time frame by setting CNY $0.765 /$ (ton $\cdot \mathrm{km}$ ) as the unit transportation cost with zero traffic flow in the link and counting the length of the link by the ranging function of Baidu Map with consideration of the variation of vehicle flow in the link.

6.3. Biobjective Pareto Optimal Path under Time-Varying Conditions. The empirical path set could be generated according to the practical path set generating strategy above.

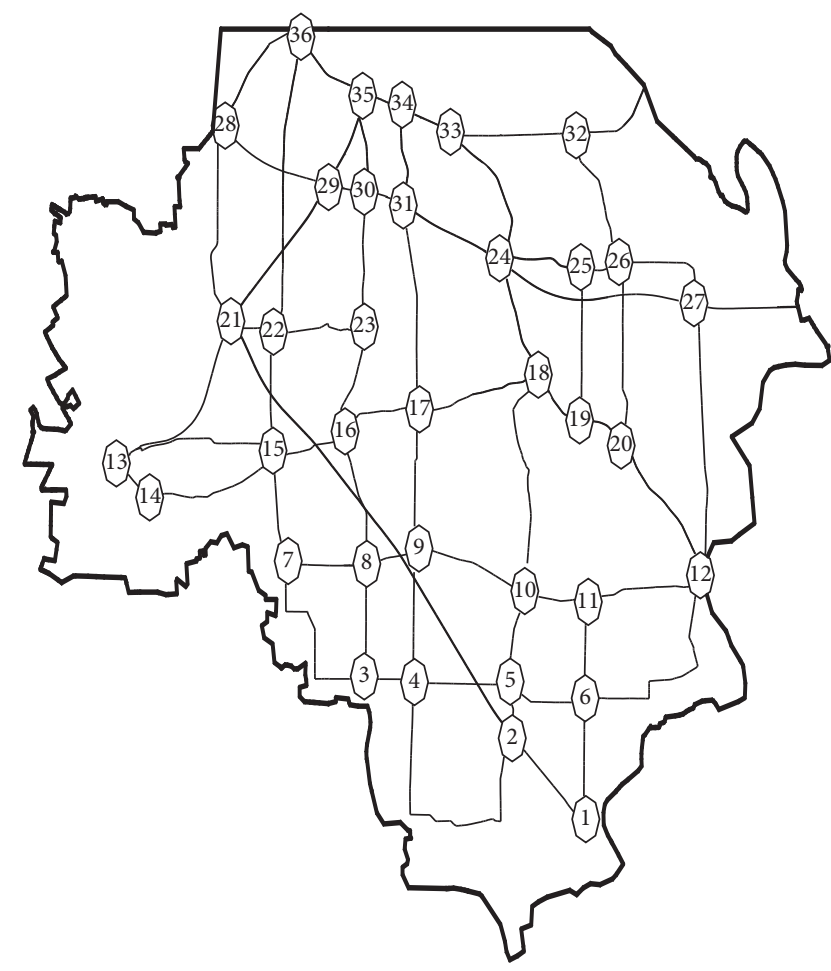

FIgure 7: Road network of City A.

Also, the risk matrix and transportation cost matrix of each time frame may be constructed, so the feasible solutions between the two points may be searched through Python programming by the improved Depth-First Search algorithm.

For example, when it is selected to output feasible solutions between node 6 and node 36 and constrain the threshold value of the number of links to be passed by each path, then the path will not pass any other node beyond the set threshold value. The starting time is set at 7:00, and the constrained quantity of links is set as $(0,12)$. There are four paths (which are demonstrated in Figure 8) in the set of nondominated solutions acquired by the depth transverse search (Figure 9 scatters diagram of feasible solutions) and rapid nondominated sorting. 


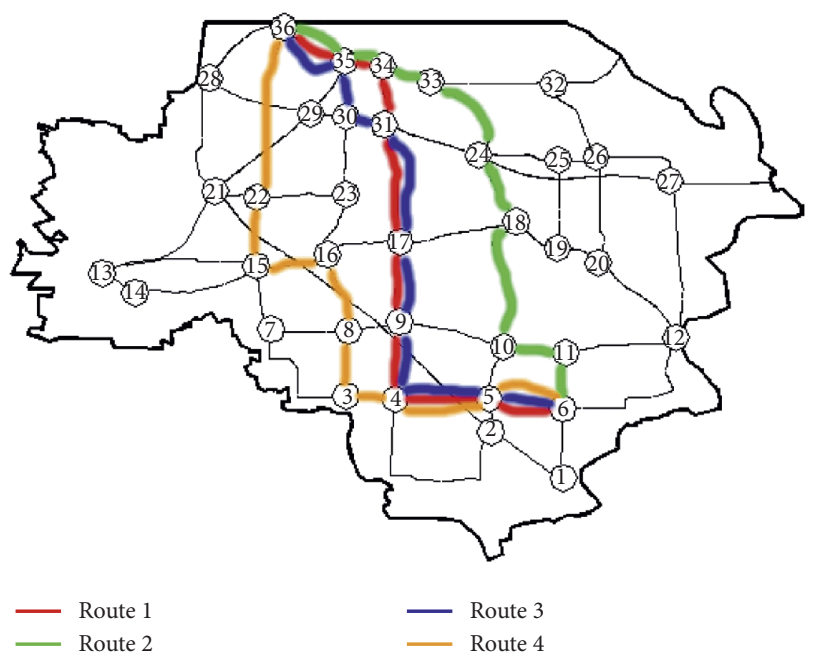

FIGURE 8: Four paths in the set of the nondominated solution.

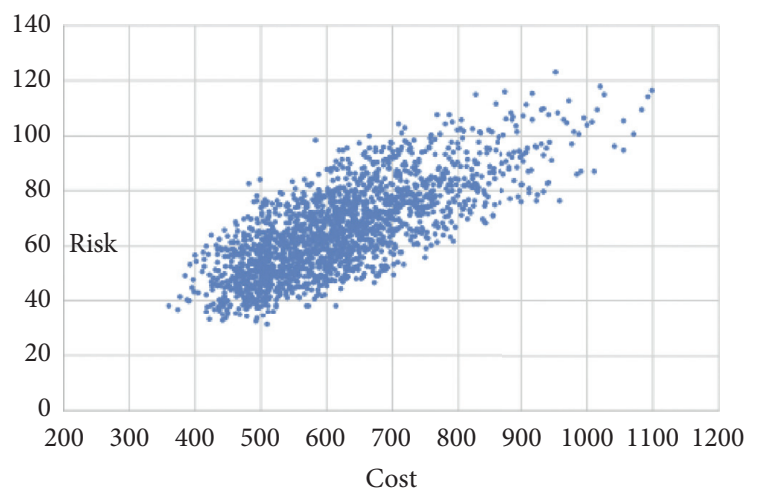

Figure 9: Scatter diagram of feasible solutions from Depth-First Search $(7: 00)$.

The set of nondominated solutions does not vary when the constrained threshold value is regulated to $(0,13),(0$, $14),(0,15)$, and $(0,16)$ for verification, demonstrating that constraint conditions will not change the generated nondominated solution set.

Similarly, the optimized path between two random points in the road network with different starting times and the Pareto nondominated solution set between some nodes with varying times of starting may be acquired, as demonstrated in Figures 10-12 and Table 7.

It is indicated in the Pareto nondominated solution set that different starting time points correspond to different Pareto optimal paths, and the same path contains different transportation costs and risks at different starting time points. The variation of transportation cost and risk of a transportation path between node 6 and node 36 at different starting time points is demonstrated in Figure 13. The decision-maker of transportation with varying preferences of expense and risk may select different starting time points and transportation paths.

Figure 13 illustrates the Pareto nondominated solution set of the transportation path from node 6 to node 36

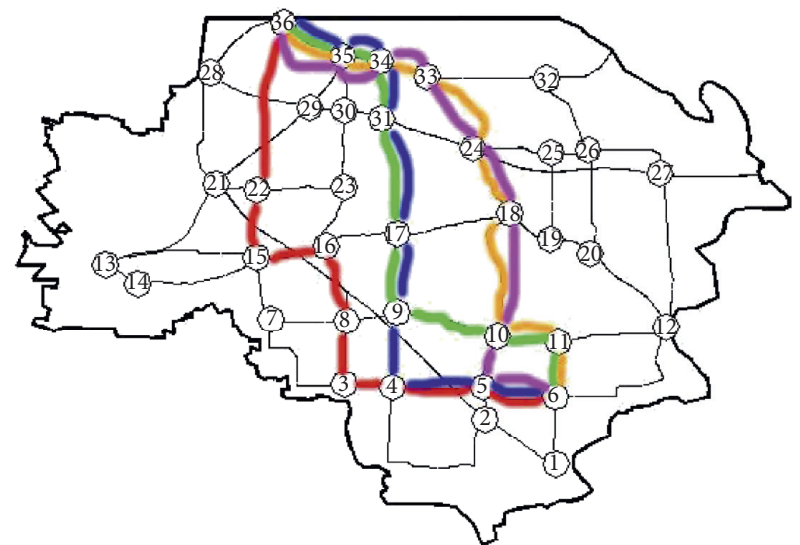

Figure 10: Pareto nondominated solutions between nodes starting at 5:00.

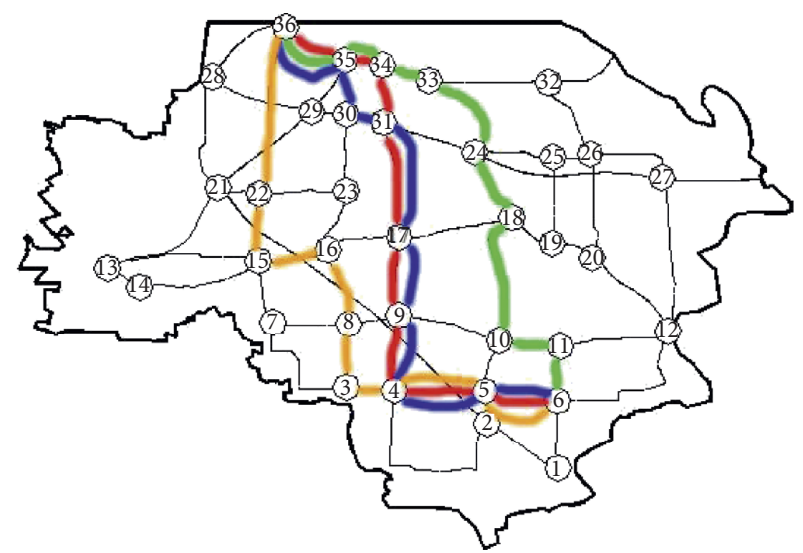

FIGURE 11: Pareto nondominated solutions between nodes starting at 7:00.

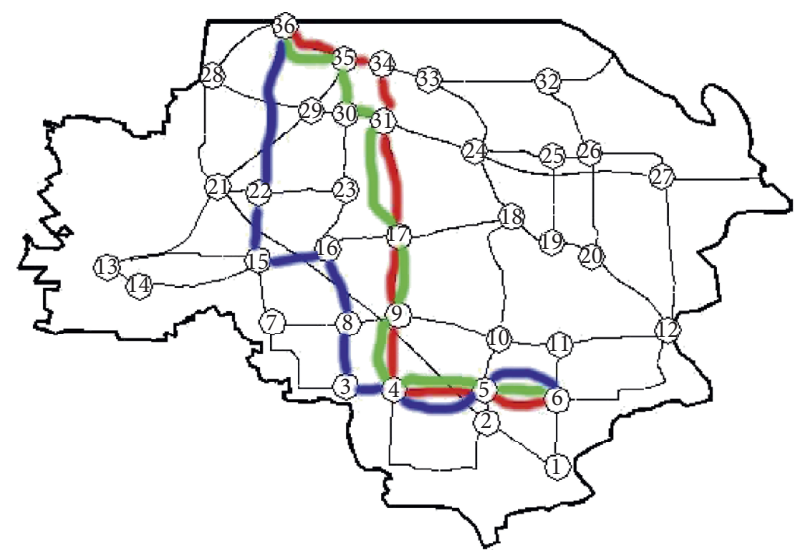

FIgURE 12: Pareto nondominated solutions between nodes starting at $8: 30$.

starting at 7:00 a.m., and altogether 4 paths are included in the set. Although the measuring unit of transportation risk and cost is the same, the results are not merely summed up as 
TABLE 7: Pareto nondominated solutions between nodes with different starting times.

\begin{tabular}{|c|c|c|c|c|c|c|}
\hline Start & End & Start time & Path & Arrival time & Risk (Yuan) & Cost (Yuan) \\
\hline \multirow{12}{*}{6} & \multirow{12}{*}{36} & \multirow{5}{*}{$5: 00$} & Red & $5: 57$ & 37.990 & 434.910 \\
\hline & & & Green & $5: 49$ & 52.980 & 376.380 \\
\hline & & & Blue & $5: 51$ & 40.360 & 387.860 \\
\hline & & & Orange & $5: 49$ & 41.620 & 377.530 \\
\hline & & & Pink & $5: 51$ & 40.130 & 390.150 \\
\hline & & \multirow{4}{*}{$7: 00$} & Red & $7: 57$ & 36.831 & 447.424 \\
\hline & & & Green & $7: 57$ & 39.321 & 439.012 \\
\hline & & & Blue & $7: 58$ & 38.231 & 443.274 \\
\hline & & & Orange & $8: 07$ & 35.386 & 510.427 \\
\hline & & \multirow{3}{*}{$8: 30$} & Red & $9: 25$ & 35.235 & 423.690 \\
\hline & & & Green & $9: 12$ & 36.017 & 417.326 \\
\hline & & & Blue & $9: 32$ & 33.776 & 475.491 \\
\hline
\end{tabular}

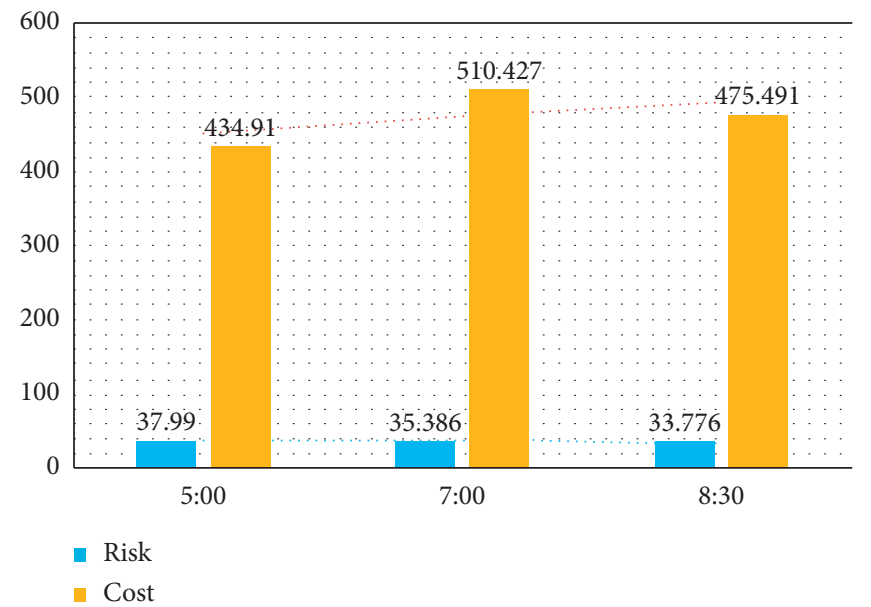

FIGURE 13: Comparison of transportation risk and cost in different time frames.

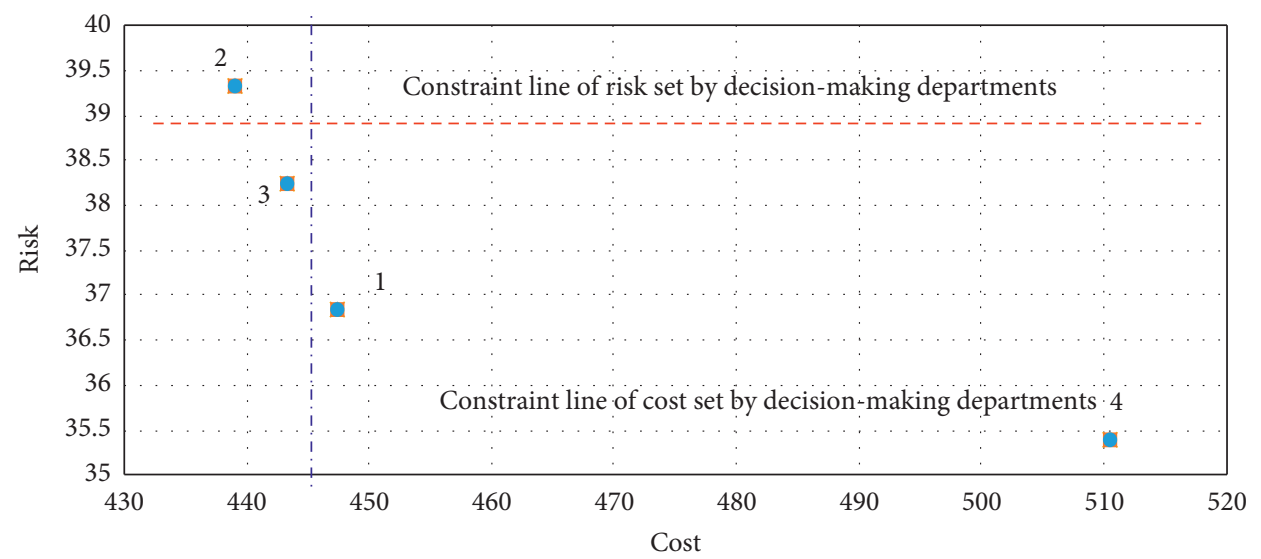

FIgURE 14: Pareto optimal path based on transportation cost and risk.

their implications differ from each other. Different decisionmakers may process these two values following the actual situation and preference of transportation.

If the imaginary line in Figure 14 is constraint conditions set by the decision-making authority on the risk and cost, paths (1), (2), and (4) may be excluded. Then, path (3) is the optimal path meeting such constraint conditions. It can be seen that the path with the minimum risk or the lowest cost is not the optimal transportation path.

\section{Conclusion}

In this paper, the authors put forward a risk assessment method of transportation path under time-varying and 
construct the biobjective path selection model to balance the transportation cost and risk based on the empirical path set for dangerous goods transportation.

A time-varying risk assessment model based on exposed population and environment-sensitive areas is proposed based on the changing law of road traffic volume and the travel law of people along the road. In this model, four kinds of correction coefficients of traffic accident probability, including road inherent characteristics, weather conditions, transportation time, and the road traffic environment, are introduced, considering the probability of accidents and the consequences of accidents, and the compensation coefficient is added to reflect the mitigation effect of emergency rescue on accident consequences.

The method for determining the scope of the accident is studied. For the determination of the scope of impact of a nonspecific accident scenario, the influence of the wind direction frequency on the diffusion range is considered, introducing the pollution coefficient. And an offset rectangular model to determine the impact area of the accident is proposed. For the leakage accident of a specific accident scenario, based on the accident point-based consequence assessment method, the equivalent impact radius is determined to determine the risk assessment scope.

Then, we apply the improved Depth-First algorithm and fast nondominated sorting to acquire the Pareto nondominated solution set between two random points in the road network for the decision-maker to select the transportation path according to the preference. At last, an example case has verified the practicability of this method.

The research results offer a practical and feasible method to reduce the threat of dangerous goods transportation to the urban road and recognize high-risk links in the mixed traffic system in advance.

\section{Data Availability}

The data used to support the findings of this study are available from the corresponding author upon request.

\section{Conflicts of Interest}

The authors declare that there are no conflicts of interest regarding the publication of this article.

\section{Acknowledgments}

This research presented in this paper was supported by the Fundamental Research Funds for the Central Universities (2020JBZD006) and the National Key R\&D Program of China (2016YFE0201700).

\section{References}

[1] B. Inanloo and B. Tansel, "A transportation network assessment tool for hazardous material cargo routing: weighing exposure health risks, proximity to vulnerable areas, delay costs and trucking expenses," Journal of Loss Prevention in the Process Industries, vol. 40, pp. 266-276, 2016.
[2] Y. Dadkar, D. Jones, and L. Nozick, "Identifying geographically diverse routes for the transportation of hazardous materials," Transportation Research Part E: Logistics and Transportation Review, vol. 44, no. 3, pp. 333-349, 2008.

[3] B. H. Dolphin, W. D. Richins, and S. R. Novascone, "Automating risk assessments of hazardous material shipments for transportation routes and mode selection," in Proceedings of the ASME International Mechanical Engineering Congress \& Exposition, Vancouver, Canada, November 2010.

[4] X. Ma, Y. Liu, and J. Lu, "Assessment of transportation risk of hazardous materials on urban roads," China Safety Science Journal, vol. 28, no. 5, pp. 170-183, 2018.

[5] I. C. L. Junior and D. A. Márcio De Almeida, "Modal choice for transportation of hazardous materials: the case of land modes of transport of bio-ethanol in Brazil," Journal of Cleaner Production, vol. 19, no. 2-3, pp. 229-240, 2011.

[6] F. Samanlioglu, "A multi-objective mathematical model for the industrial hazardous waste location-routing problem," European Journal of Operational Research, vol. 226, no. 2, pp. 332-340, 2013.

[7] D. Pamučar, S. Ljubojević, D. Kostadinović, and B. Đorović, "Cost and risk aggregation in multi-objective route planning for hazardous materials transportation-a neuro-fuzzy and artificial bee colony approach," Expert Systems with Applications, vol. 65, pp. 1-15, 2016.

[8] A. Mahmoudabadi and L. Zhang, "Two chaotic patterns of dynamic risk definition for solving hazardous materials routing problem," Journal of Engineering, vol. 2015, Article ID 905864, 6 pages, 2015.

[9] C. Kwon, "Conditional value-at-risk model for hazardous materials transportation," in Proceedings of the 2011 Winter Simulation Conference (WSC), pp. 1703-1709, IEEE, Phoenix, AZ, USA, December 2011.

[10] J. Jassbi and P. Makvandi, "Route selection based on soft MODM framework in transportation of hazardous materials," Applied Mathematical Sciences, vol. 63, no. 4, pp. 3121-3132, 2010.

[11] N. Haghighi, X. C. Liu, G. Zhang, and R. J. Porter, "Impact of roadway geometric features on crash severity on rural twolane highways," Accident Analysis \& Prevention, vol. 111, pp. 34-42, 2018.

[12] Q. Zeng, Q. Guo, S. C. Wong, H. Wen, H. Huang, and X. Pei, "Jointly modeling area-level crash rates by severity: a bayesian multivariate random-parameters spatio-temporal Tobit regression," Transportmetrica A: Transport Science, vol. 15, no. 2, pp. 1867-1884, 2019.

[13] Q. Zeng, W. Hao, J. Lee, and F. Chen, "Investigating the impacts of real-time weather conditions on freeway crash severity: a bayesian spatial analysis," International Journal of Environmental Research and Public Health, vol. 17, no. 8, p. 2768, 2020.

[14] Q. Zeng, H. Wen, S. C. Wong, H. Huang, Q. Guo, and X. Pei, "Spatial joint analysis for zonal daytime and nighttime crash frequencies using a bayesian bivariate conditional autoregressive model," Journal of Transportation Safety \& Security, vol. 12, no. 4, pp. 566-585, 2020.

[15] M. Imprialou and M. Quddus, "Crash data quality for road safety research: current state and future directions," Accident Analysis \& Prevention, vol. 130, pp. 84-90, 2019.

[16] R. Pradhananga, E. Taniguchi, T. Yamada, and A. G. Qureshi, "Bi-objective decision support system for routing and scheduling of hazardous materials," Socio-Economic Planning Sciences, vol. 48, no. 2, pp. 135-148, 2014. 
[17] X. Wang and H. Wang, "Driving behavior clustering for hazardous material transportation based on genetic fuzzy c-means algorithm," IEEE Access, vol. 8, pp. 11289-11296, 2020.

[18] K. Liu, Research on diffusion model and risk assessment of toxic gas leakage in road transportation by tank trucks, Ph.D. thesis, Chang'an University, Xi'an, China, 2011.

[19] Z. Zhou, Y. Cai, R. Ke, and J. Yang, "A collision avoidance model for two-pedestrian groups: considering random avoidance patterns," Physica A: Statistical Mechanics and Its Applications, vol. 475, pp. 142-154, 2017.

[20] F. Chen, M. Song, and X. Ma, "Investigation on the injury severity of drivers in rear-end collisions between cars using a random parameters bivariate ordered probit model," International Journal of Environmental Research and Public Health, vol. 16, no. 14, p. 2632, 2019.

[21] F. Chen, S. Chen, X. Ma, and X. Ma, "Crash frequency modeling using real-time environmental and traffic data and unbalanced panel data models," International Journal of Environmental Research and Public Health, vol. 13, no. 6, p. 609, 2016.

[22] Z. Wu, C. Ren, and D. Yingquan, Risk Assessment Method of Road Transportation Accident of Hazardous Materials, Chemical Industry Press, Beijing, China, 2014.

[23] B. Dong, X. Ma, F. Chen, and S. Chen, "Investigating the differences of single- and multi-vehicle accident probability using mixed logit model," Journal of Advanced Transportation, vol. 2018, Article ID 2702360, 9 pages, 2018.

[24] D. Ma, X. Song, and P. Li, "Daily traffic flow forecasting through a contextual convolutional recurrent neural network modeling inter-and intra-day traffic patterns," IEEE Transactions on Intelligent Transportation Systems, Online., 2020.

[25] H. Guo and M. Chen, "Short-term effect of air pollution on asthma patient visits in Shanghai area and assessment of economic costs," Ecotoxicology and Environmental Safety, vol. 161, pp. 184-189, 2018.

[26] C. Zhang, C. Xiao, J. Chen et al., "Consequence and risk assessment of chemical leakage accident concerning emergency rescue," Journal of Tsinghua University, vol. 49, no. 5, pp. 621-624, 2009.

[27] E. Erkut and O. Alp, "Designing a road network for hazardous materials shipments," Computers \& Operations Research, vol. 34, no. 5, pp. 1389-1405, 2007.

[28] E. Erkut and F. Gzara, "Solving the hazmat transport network design problem," Computers \& Operations Research, vol. 35, no. 7, pp. 2234-2247, 2008.

[29] D. Ma, J. Xiao, X. Song, X. Ma, and S. Jin, “A back-pressurebased model with fixed phase sequences for traffic signal optimization under oversaturated networks," IEEE Transactions on Intelligent Transportation Systems, 2020.

[30] S. Feng, Traffic Graph Theory, China Communications Press Co., Ltd., Beijing, China, 2017.

[31] G. Chen, "Analysis of influence of different freight settlement modes upon transportation efficiency of petroleum products," Modern Industrial Economy and Informationization, vol. 9, no. 4, pp. 18-21, 2014. 